\title{
Síndrome de burnout em profissionais de enfermagem no ambiente hospitalar: uma revisão integrativa
}

\author{
Burnout syndrome in nursing professionals in the hospital environment: an integrating \\ review
}

Síndrome de burnout en profesionales de enfermería en el medio ambiente hospitalario: una revisión integrativa

Frederico Marques Andrade ${ }^{1 *}$, Lanuza Borges Oliveira ${ }^{1,2}$, Maria Cecília Dias Corrêa ${ }^{2}$, Camila Bretas Santos ${ }^{3}$, Julia de Oliveira e Silva ${ }^{4}$, Luiz Fernando Abasse Maciel ${ }^{2}$, Rafael Jose Capuchinho Rocha $^{5}$, Ícaro Amorim Monteiro ${ }^{1}$, Daniela Oliveira Lima Magalhães ${ }^{1}$, Tatiane Amélia Ferreira ${ }^{2}$, Renato Martins Tolentino'.

\section{RESUMO}

Objetivo: Este estudo objetiva revisar a Síndrome de Burnout, os fatores que a determinam e as consequências para a saúde dos profissionais de enfermagem. Métodos: Trata-se de uma pesquisa de revisão integrativa, desenvolvida com base em material já publicado, principalmente artigos publicados em periódicos nacionais e internacionais. Resultados: Os resultados revelaram que a Síndrome de Burnout é um mal que atinge um número significativo de profissionais de enfermagem, que sofrem de reiteradas exposições ao estresse e aos fatores determinantes da doença. Dentre os fatores para o desenvolvimento da Síndrome de Burnout entre enfermeiros, pode-se destacar o ambiente organizacional, a sobrecarga de trabalho, o grande controle sobre as tarefas e condições individuais. Conclusão: Acredita-se ser necessário que os futuros estudos visem identificar ações preventivas, contribuindo para redução das ocorrências da Síndrome de Burnout entre os enfermeiros.

Palavras-chaves: Esgotamento profissional, Gestão em Saúde, Saúde do trabalhador.

\begin{abstract}
Objective: This study aims to review Burnout Syndrome, the factors that determine it and the health consequences of nursing professionals. Methods: This is an integrative review research, developed based on material already published, mainly articles published in national and international journals. Results: The results revealed that Burnout Syndrome is an illness that reaches a significant number of nursing professionals, who suffer from repeated exposures to stress and the determinants of the disease. Among the factors for the development of Burnout Syndrome among nurses, we can highlight the organizational environment, work overload, great control over individual tasks and conditions. Conclusion: It is believed that it is necessary that future studies aim to identify preventive actions, contributing to reduce the occurrence of Burnout Syndrome among nurses.
\end{abstract}

Keywords: Occupational exhaustion, Health Management, Worker's health.

1 Universidade Estadual de Montes Claros (Unimontes), Montes Claros-MG.

*E-mail: frederico.marques@unimontes.br

2 Faculdades Integradas Pitágoras (FIP-MOC), Montes Claros-MG

${ }^{3}$ Universidade FUMEC, Belo Horizonte-MG.

${ }^{4}$ Faculdade Governador Ozanam Coelho, Ubá-MG.

${ }^{5}$ Faculdades Unidas do Norte de Minas (FUNORTE) Montes Claros-MG 


\section{RESUMEN}

Objetivo: Este estudio objetiva revisar el Síndrome de Burnout, los factores que la determinan y las consecuencias para la salud de los profesionales de enfermería. Métodos: Se trata de una investigación de revisión integrativa, desarrollada con base en material ya publicado, principalmente artículos publicados en periódicos nacionales e internacionales. Resultados: Los resultados revelaron que el Síndrome de Burnout es un mal que alcanza un número significativo de profesionales de enfermería, que sufren de reiteradas exposiciones al estrés ya los factores determinantes de la enfermedad. Entre los factores para el desarrollo del Síndrome de Burnout entre enfermeros, se puede destacar el ambiente organizacional, la sobrecarga de trabajo, el gran control sobre las tareas y condiciones individuales. Conclusión: Se cree necesario que los futuros estudios busquen identificar acciones preventivas, contribuyendo a la reducción de las ocurrencias del Síndrome de Burnout entre los enfermeros.

Palabras claves: Agotamiento profesional, Gestión en Salud, Salud del trabajador.

\section{INTRODUÇÃO}

Mesmo com toda a modernidade que cerca o cotidiano das empresas, a saúde dos trabalhadores sofrem ainda fortes pressões físicas e psicológicas. Contínuas e crescentes evoluções técnicas, além de transformações no modo de produção, exigem do indivíduo maior tempo na preparação e qualificação profissional, pressionando ainda mais por resultados. Os profissionais da área da saúde ganham destaque por estarem expostos em seu cotidiano à situações estressantes e a vários riscos ocupacionais especialmente a equipe de Enfermagem (SOUSA MVH, 2012).

Neste leque de situações, várias síndromes podem ser desenvolvidas, destaque para a a Síndrome de Burnout. Essa síndrome, por sua vez, é uma doença laboral caracterizada pelo desgaste do profissional em seu ambiente de trabalho, evidenciado pela desmotivação e insatisfação dos trabalhadores. Está diretamente ligada aos profissionais que estão em contato direto com pessoas, tais como: profissionais de saúde em geral e professores (LIMA FD, et al., 2007).

Para Carlotto MS e Câmara SG (2008), suas principais manifestações clínicas são sintomas físicos e psíquicos, onde os profissionais acabam perdendo o interesse pelo trabalho e vida social. Este processo acontece de forma gradual de desgaste emocional e desmotivação com o serviço. O termo Burnout surgiu na década de 50 para a descrição do caso de uma enfermeira psiquiátrica desiludida com a sua rotina de trabalho. O caso ficou conhecido como "Miss Jones". Em 1960, Graham Greene publica o relato do caso de um arquiteto que abandonou a sua profissão devido a sentimentos de desilusão com o seu ofício (CARLOTTO MS, 2002).

Como consequência de vários relatos de caso, diversos estudos analisaram o estresse entre profissionais da área de saúde, com destaque para a enfermagem. A enfermagem é considerada uma profissão com peculiaridades que favorecem a síndrome de Burnout, graças ao número de horas em permanência da atividade, a continua necessidade de mediação nas relações entre pacientes, familiares e técnicos, associados a atividades administrativas e gerenciais (GUIDO LA, et al., 2012).

Diante tal exposto, o problema proposto para a realização do presente estudo considera os elementos geradores do estresse laboral os quais, a equipe de enfermagem esta submetida e que propiciam grande probabilidade para o surgimento desta síndrome: alto nível de exaustão profissional, sobrecarga de atribuições, salário incompatível, conflito com colegas e/ou instituição, além de lidarem diretamente com a morte e estarem vulneráveis ao risco de contaminação e violência (SOUSA MVH, 2012).

Assim, o presente estudo busca revisar as causas e consequências da Síndrome de Burnout para a saúde dos profissionais de enfermagem no ambiente hospitalar, com intuito de contribuir para adoção de medidas efetivas na prevenção desta síndrome, promovendo a saúde no trabalho e qualidade de vida para estes profissionais e consequentemente diminuindo o absenteísmo no trabalho. 


\section{MÉTODOS}

Trata-se de uma pesquisa de Revisão Integrativa, de caráter descritivo e exploratório. Tem por finalidade reportar e avaliar o conhecimento produzido em pesquisas prévias, destacando conceitos, procedimentos, resultados, discussões e conclusões relevantes para seu trabalho acerca da Síndrome de Burnout e suas principais causas e consequências para a saúde dos profissionais de enfermagem.

Para a coleta de dados, foram realizadas pesquisas científicas através de um levantamento bibliográfico, no período de 2006 a 2016, em periódicos de enfermagem nacionais e internacionais, este período, a partir de 2006, justificado pelo aumento exponencial de profissionais de enfermagem no mercado, também justificados ao fato de terem sido considerados os de maior circulação pelos autores do presente estudo.

A busca foi realizada por meio da consulta ao site de busca da Biblioteca virtual de saúde (BVS) utilizando a base de dados a MEDLINE (Medical Literature Analysis and Retriveal System Online); LILACS (Base de dados da Literatura Latino-Americana em Ciência da Saúde) e a BDENF (Biblioteca de Enfermagem). Os critérios de inclusão foram: artigos que enquandrassem ao corte temporal, anteriormente descrito, estudos que respondessem ao objetivo do trabalho e estudos com resultados de pesquisas brasileiras. Como resultado, somente foram encontrados artigos na língua portuguesa. Foram utilizados os descritores associados "Bournout" ou "Burnout" e "enfermagem". Na primeira busca surgiram 87 artigos, filtrando por artigos disponíveis obteve-se 56, após a leitura, filtrando para o objetivo da pesquisa, ficaram 13 artigos.

\section{RESULTADOS E DICUSSÃO}

\section{Síndrome de Burnout}

Segundo Trigo TR et al. (2007) a Síndrome de Burnout também é conhecida como Síndrome do esgotamento profissional é um distúrbio psíquico descrito pela primeira vez em 1974 por Freudenberger, médico americano, que a definiu como uma doença que agride a sociedade de forma muito rápida e assustadora.

O conjunto de sinais e sintomas relacionados às circunstâncias de estresse laboral crônico, numa tríade que levam a Síndrome de Burnout: o cansaço emocional, a despersonalização e a falta de realização pessoal. Manifestam-se em pessoas sem antecedentes de distúrbios psicopatológicos e associados à exaustão mental e emocional, fadiga e depressão (CARLOTTO MS, 2002).

Tem maior relevância nos sintomas comportamentais e mentais na comparação com os sintomas físicos, assim, a Síndrome de Burnout, nos profissionais de saúde, emerge como um alerta a situações crônicas de estresse e às extensas jornadas de trabalho, afetando a produção, relacionamentos sociais, qualidade de vida, desempenho nas organizações onde atua (FRANÇA FM, et al., 2012).

Para França FM et al. (2012), a Síndrome de Burnout envolve três componentes que aparecem ligados porém são independentes - exaustão emocional, despersonalização e baixa realização pessoal no trabalho, são eles: Exaustão emocional tem-se uma ausência de energia e a sensação de esgotamento físico e emocional; Despersonalização é percebida pela indiferença diante das relações sociais e com os pacientes o trabalhador parece não se envolver com o trabalho; Baixa realização no trabalho é percebida principalmente pelas frequentes faltas ao trabalho, sensação de insuficiência, desmotivação e baixa auto estima.

Assim, a Síndrome de Burnout é considerada a partir de grandes exposições de estresse no ambiente de trabalho gerando um esgotamento físico e mental. Esta é uma doença que ocorre no sistema psíquico do trabalhador no qual se destaca a enfermagem, resultado este, decorrente de situações de estresse, graças as relações intensas de trabalho e suas expectativas com relação ao seu desenvolvimento profissional, 
quanto sua dedicação e a desilusão de não alcançarem o objetivo esperado (JODAS DA e HADDAD MCL, 2009).

Tabela 1 - Artigos selecionados sobre Síndrome de Burnout para a pesquisa bibliográfica - 2018

\begin{tabular}{lllll}
\hline Título & Ano & Periódico & Participantes \\
\hline $\begin{array}{l}\text { 1. Análise da produção científica sobre a } \\
\text { Síndrome de Burnout no Brasil }\end{array}$ & 2008 & $\begin{array}{l}\text { Programa de Pós- } \\
\text { Graduação em Psicologia }\end{array}$ & Psicólogos \\
$\begin{array}{l}\text { 2. Prevalência da Síndrome de Burnout em } \\
\text { enfermeiros atuantes em UTI }\end{array}$ & 2013 & $\begin{array}{l}\text { Psicologia.pt } \\
\text { O Portal dos Psicólogos }\end{array}$ & $\begin{array}{l}\text { Psicólogo e } \\
\text { Enfermeiros }\end{array}$
\end{tabular}

3. Burnout and labour aspects in the nursing 2012 Revista Latino -Americana Enfermeiros teams at two medium-sized hospitals de Enfermagem

4. Impacto do stress ocupacional e Burnout 2010 para enfermeiros

Revista eletrônica Enfermeiros semestral de enfermeira -

Enfermeria Global

5. Estresse e Burnout entre residentes

2012 Revista Latino -Americana Enfermeiros multiprofissionais de Enfermagem

6. Síndrome de Burnout em trabalhadores de 2009 enfermagem de um pronto socorro de hospital universitário

Acta Paulista de Enfermeiros

Enfermagem

2007

Revista Brasileira de

Médicos

7. Síndrome de Burnout em Residentes da Universidade Federal de Uberlândia Educação Médica

8. Burnout e estresse los Enfermeiros de hum Hospital Universitário de Alta

2010 Revista Latino -Americana Enfermeiros e Médico Complexidade de Enfermagem

9. Síndrome de Burnout em profissionais de 2009 Revista Acervo Saúde Psicólogos saúde: uma revisão bibliográfica

10. Prevalência da síndrome de burnout em

2009

Caderno de Saúde Publica Médicos trabalhadores de enfermagem de um hospital de grande porte da Região Sul do Brasil

11. Síndrome de Burnout e absenteísmo em enfermeiros no contexto hospitalar:

2012 Comunicação em Ciências Enfermeiros da Saúde revisão integrativa da literatura brasileira

12. Síndrome de Burnout em profissionais da saúde: estudo bibliográfico

2012 Monografia Enfermeiro

13. Síndrome de burnout ou estafa

2007 Revista de psiquiatria Médicos

profissional e os transtornos psiquiátricos clinica

Fonte: dados da pesquisa, 2018. 
Tabela 2 - Análise inicial dos artigos selecionados sobre Síndrome de Burnout para a pesquisa bibliográfica - 2018

\begin{tabular}{|c|c|c|}
\hline Título & $\begin{array}{l}\text { Modalidade de } \\
\text { pesquisa }\end{array}$ & Síntese dos resultados \\
\hline $\begin{array}{l}\text { 1. Análise da produção científica } \\
\text { sobre a Síndrome de } \\
\text { Burnout no Brasil }\end{array}$ & $\begin{array}{l}\text { Pesquisa } \\
\text { bibliográfica na base } \\
\text { de dados BVS. }\end{array}$ & $\begin{array}{l}\text { Necessidade de construção de } \\
\text { uma agenda de pesquisa sobre } \\
\text { Burnout, conferindo maior } \\
\text { relevância ao tema. }\end{array}$ \\
\hline $\begin{array}{l}\text { 2. Prevalência da Síndrome de } \\
\text { Burnout em enfermeiros atuantes } \\
\text { em UTI }\end{array}$ & $\begin{array}{l}\text { Revisão } \\
\text { Bibliográfica }\end{array}$ & $\begin{array}{l}\text { Condições de trabalho enfrentadas } \\
\text { sendo o principal desencadeante o } \\
\text { estresse relacionado a tais } \\
\text { condições }\end{array}$ \\
\hline $\begin{array}{l}\text { 3. Burnout and labour aspects in } \\
\text { the } \\
\text { nursing teams at two medium- } \\
\text { sized } \\
\text { hospitals }\end{array}$ & $\begin{array}{l}\text { Estudo } \\
\text { epidemiológico } \\
\text { quantitativo } \\
\text { descritivo, do tipo } \\
\text { transversal }\end{array}$ & $\begin{array}{l}\text { Revelou a necessidade de se } \\
\text { propor mudanças organizacionais } \\
\text { no ambiente de trabalho, a fim } \\
\text { de diminuir a interferência desses } \\
\text { fatores na saúde do trabalhador }\end{array}$ \\
\hline $\begin{array}{l}\text { 4. Impacto do stress ocupacional } \\
\text { e Burnout para enfermeiros }\end{array}$ & $\begin{array}{l}\text { Levantamento } \\
\text { Bibliográfico em } \\
\text { bases de dados } \\
\text { eletrônicos }\end{array}$ & $\begin{array}{l}\text { Verifica que há uma escassez de } \\
\text { estudos e análises de } \\
\text { intervenções para redução do } \\
\text { estresse ocupacional e o burnout } \\
\text { que sejam exequíveis dentro do } \\
\text { turbulento ambiente hospitalar }\end{array}$ \\
\hline $\begin{array}{l}\text { 5. Estresse e Burnout entre } \\
\text { residentes multiprofissionais }\end{array}$ & $\begin{array}{l}\text { Estudo Analítico, } \\
\text { Transversal, } \\
\text { Quantitativo }\end{array}$ & $\begin{array}{l}\text { Sugere-se a realização de novos } \\
\text { estudos analíticos sobre estresse } \\
\text { e Burnout entre profissionais da } \\
\text { área da saúde, com vistas à } \\
\text { produção de evidências científicas } \\
\text { sobre a temática }\end{array}$ \\
\hline $\begin{array}{l}\text { 6. Síndrome de Burnout em } \\
\text { trabalhadores de enfermagem de }\end{array}$ & $\begin{array}{l}\text { Estudo descritivo } \\
\text { quantitativo }\end{array}$ & $\begin{array}{l}\text { A dinâmica organizacional de um } \\
\text { Pronto Socorro gera uma } \\
\text { sobrecarga e tensão }\end{array}$ \\
\hline $\begin{array}{l}\text { pronto socorro de hospital } \\
\text { universitário }\end{array}$ & & $\begin{array}{lll}\text { ocupacional sendo necessário } \\
\text { desenvolver estratégias de } \\
\text { reorganização do processo de } \\
\text { trabalho diminuindo fontes de } \\
\text { estresse }\end{array}$ \\
\hline $\begin{array}{l}\text { 7. Síndrome de Burnout em } \\
\text { Residentes da Universidade } \\
\quad \text { Federal } \\
\text { de Uberlândia }\end{array}$ & Analise Estátistica & $\begin{array}{l}\text { Suscita a preocupação e a } \\
\text { necessidade de intervenções } \\
\text { preventivas e curativas }\end{array}$ \\
\hline
\end{tabular}




\begin{tabular}{|c|c|c|c|}
\hline & $\begin{array}{l}\text { 8. Burnout e estresse los } \\
\text { Enfermeiros de hum Hospital } \\
\text { Universitário de Alta } \\
\quad \text { Complexidade }\end{array}$ & $\begin{array}{l}\text { Estudo Transversal, } \\
\text { Exploratório, } \\
\text { Analítico e } \\
\text { Correlacional }\end{array}$ & $\begin{array}{l}\text { Existe importante vulnerabilidade } \\
\text { dos enfermeiros para Burnout } \\
\text { potencializada pela vivência de } \\
\text { estresse no ambiente de trabalho } \\
\text { hospitalar }\end{array}$ \\
\hline & $\begin{array}{l}\text { 9. Síndrome de Burnout em } \\
\text { profissionais de saúde: uma } \\
\text { revisão } \\
\text { bibliográfica }\end{array}$ & $\begin{array}{l}\text { Levantamento } \\
\text { Bibliográfico nas } \\
\text { bases de dados: } \\
\text { IndexPsi, Scielo, } \\
\text { Lllacs e Psyclnfo }\end{array}$ & $\begin{array}{l}\text { É importante notar que a dinâmica } \\
\text { organizacional do trabalho em } \\
\text { hospitais gera grande tensão } \\
\text { ocupacional, sendo necessário } \\
\text { monitorar periodicamente a saúde } \\
\text { mental e física desses } \\
\text { trabalhadores }\end{array}$ \\
\hline & $\begin{array}{l}\text { 10. Prevalência da síndrome de } \\
\text { burnout em trabalhadores de } \\
\text { enfermagem de um hospital } \\
\text { de grande porte da Região } \\
\text { Sul do Brasil }\end{array}$ & $\begin{array}{l}\text { Abordagem } \\
\text { Quantitativa }\end{array}$ & $\begin{array}{l}\text { Outros estudos devem continuar } \\
\text { desvendando as relações entre o } \\
\text { trabalho e a saúde dos } \\
\text { trabalhadores }\end{array}$ \\
\hline & $\begin{array}{l}\text { 11. Síndrome de Burnout e } \\
\text { absenteísmo em enfermeiros } \\
\text { no contexto hospitalar: } \\
\text { revisão integrativa da } \\
\text { literatura brasileira }\end{array}$ & $\begin{array}{l}\text { Revisão Integrativa } \\
\text { nas bases de dados } \\
\text { BDENF, MedLine, } \\
\text { Lilacs e Scielo }\end{array}$ & $\begin{array}{l}\text { Tais fatores devem ser avaliados } \\
\text { do ambiente ocupacional, a fim de } \\
\text { estabelecer o diagnóstico } \\
\text { situacional e interferir nos fatores } \\
\text { determinantes, visando melhoria } \\
\text { da qualidade de vida dos } \\
\text { enfermeiros }\end{array}$ \\
\hline & $\begin{array}{l}\text { 12. Síndrome de Burnout em } \\
\text { profissionais da saúde: } \\
\text { estudo bibliográfico }\end{array}$ & $\begin{array}{l}\text { Estudo Bibliográfico } \\
\text { nas bases de dados } \\
\text { Lilacs e MedLine }\end{array}$ & $\begin{array}{l}\text { É imprescindível a implementação } \\
\text { de atividades voltadas para } \\
\text { prevenção e promoção da saúde } \\
\text { dos trabalhadores, amenizando o } \\
\text { risco de desenvolvimento da } \\
\text { Síndrome de Burnout }\end{array}$ \\
\hline 13. & $\begin{array}{l}\text { Síndrome de burnout ou estafa } \\
\text { profissional e os transtornos } \\
\text { psiquiátricos }\end{array}$ & $\begin{array}{l}\text { Revisão } \\
\text { Bibliográfica nas } \\
\text { bases de dados } \\
\text { MedLine, Scielo }\end{array}$ & $\begin{array}{l}\text { A prevalência do Burnout nos } \\
\text { vários países ainda é incerta, mas } \\
\text { dados apontam acometimento } \\
\text { significativo que justifica mais } \\
\text { estudos a respeito dessa patologia } \\
\text { com fatores de risco multifatoriais } \\
\text { (indivíduo, trabalho, organização) }\end{array}$ \\
\hline
\end{tabular}

Fonte: dados da pesquisa, 2018.

\section{Prevalência da Síndrome de Burnout no Brasil}

Duarte AP et al. (2013), em pesquisa sobre a Síndrome de Burnout entre enfermeiros de uma unidade hospitalar, fizeram uma revisão bibliográfica onde apontaram que $59,4 \%$ dos entrevistados encontravam-se estressados e assim com maiores chances de desenvolverem Burnout.

O estudo de Moreira DS et al. (2009) mostrou a prevalência da Síndrome de Burnout em trabalhadores de enfermagem de um hospital de grande porte da Região Sul do Brasil. O grupo de trabalhadores era 
composto em sua maioria por mulheres, entre 26 e 35 anos e com menos de dez anos de profissão. A prevalência da Síndrome de Burnout encontrado foi considerado alto, pois foi encontrada em mais de um terço deles $-37,5 \%$.

Neste contexto, França FM et al. (2012) identificaram que os profissionais de enfermagem mais acometidos pela Síndrome de Burnout eram aqueles com regime de trabalho diarista, com carga horária semanal de 30 horas, vínculo empregatício por meio de contrato, duplo vínculo empregatício, menor tempo de formação, com menos de um ano de trabalho na unidade e aqueles que possuíam, como setor de lotação, cargo administrativo.

\section{Principais causas e consequências da Síndrome de Burnout entre os profissionais da enfermagem}

O cotidiano do profissional de enfermagem no cuidar provoca tensão emocional permanente, atenção e muita responsabilidade. Esta é a principal característica do trabalho em saúde, pois lida com a dor, o sofrimento e a morte, afetando os trabalhadores de instituições hospitalares, favorecendo o aparecimento da Síndrome de Burnout (FRANÇA FM, et al., 2012).

Aspectos organizacionais, condições inadequadas de trabalho, jornadas prolongadas, excesso de tarefas, conflitos interpessoais, baixa autonomia e de remuneração, associados à sobrecarga psicológica, cognitiva e física dos profissionais (REZENDE R, et al., 2012). O estresse entre os profissionais de saúde pode estar relacionado à administração da responsabilidade profissional, a assistência há pacientes em estado critico, conflito de situações problemáticas, controle do volume de conhecimento e do estabelecimento dos limites de sua identidade pessoal e profissional (GUIDO LA, et al., 2012).

O estresse é considerado como um estado prematuro ou real de atenção ao equilíbrio do organismo e a reação deste organismo a essa ameaça, buscando devolver o equilíbrio por meio de um conjunto de respostas fisiológicas e comportamentais. A resposta ao estresse precisa estar na mesma medida da ameaça isto faz com que mobilize energia suficiente para que o organismo se reequilibre. Quando essa energia é maior ou insuficiente, o estresse atinge o corpo produzindo efeitos sobre a digestão, 0 crescimento, a reprodução, dentre outros (RIBEIRO RP, et al. 2018). Nesse contexto, a exposição a situações estressoras frequentes, relacionadas às condições de trabalho, propicia se então a Síndrome de Burnout. O estresse no trabalho, quando ultrassa níveis suportáveis, torna-se Burnout. A palavra Burnout é um termo que significa esgotamento, algo ou alguém não possui mais energias para produzir, chegou ao limite das forças, não funciona mais, queimou até o final (GRAZZIANO ES e BIANCHI ERF, 2010).

Ainda segundo os autores supracitados, a pessoa acometida pelo Burnout tem vários aspectos de sua vida afetados: Saúde física: fadiga crônica, exaustão, gripes constantes, perdas de consciência momentânea, dor de cabeça frequentes, dor muscular, hipertensão arterial, imunodeficiência, distúrbios cardiovasculares, gastrintestinais, respiratórios, sexuais e do sono; Saúde cognitiva e emocional: dificuldade de concentração e de atenção, pouca memória, mau humor, auto isolamento, impaciência, labilidade emocional, baixa auto-estima, ansiedade, depressão, vulnerabilidade, medo: Comportamento: frustração, impaciência, hostilidade, intolerância, rigidez, rigor excessivo, perda de iniciativa, questiona do próprio julgamento, trabalho com pouco rendimento (GRAZZIANO ES e BIANCHI ERF, 2010).

A Síndrome de Burnout entre os enfermeiros também prejudica as instituições de saúde já que o absenteísmo é alto, além de ser prejudicial individualmente também atinge a categoria profissional, devido a baixa qualidade na assistência de enfermagem oferecida aos pacientes e familiares, nos serviços de saúde, quando se privilegia a humanização da assistência à saúde (LORENZ VR, et al., 2010).

\section{Fatores determinantes para o desenvolvimento da Síndrome de Burnout entre os profissionais de enfermagem}

As principais fontes de estresse associados ao diagnóstico de Burnout na enfermagem, estão relacionados a recorrentes fatores: ausência de reconhecimento do trabalho, dificuldades na rotina, erros na 
coordenação da equipe de trabalho, ausência de serviços de auxílio ao profissional, ineficiência da estrutura administrativa no serviço de saúde, pouco ou nenhum apoio social, baixa remuneração em relação ao esforço empregado, inexistência de oportunidades para desenvolvimento pessoal, sobrecarga de trabalho, entre outros (MATUBARO KCA, et al., 2009).

Neste Contexto, Trigo TR et al. (2007) afirmam que os principais fatores determinantes para o desenvolvimento da Síndrome de Burnout entre os profissionais, são os organizacionais, individuais, laborais e sociais. Os organizacionais referem-se a burocracia do serviço, normas, mudanças na empresa e as relações hierarquicas nela existentes. Os fatores individuais referem-se a personalidade do trabalhador assim com o o modo como ele lida com essas mudanças emocionais dentro da empresa. Os laborais estão relacionados com a sobrecarga, o controle perante suas atividades, as observações perante as atividades realizadas na empresa. Os fatores sociais relacionam-se com o suporte social e familiar e seu reflexo no meio familiar e social do indivíduo, influenciado pelos seus valores e normais culturais.

Além disso, vale enfatizar que a prevalência de Burnout era menor, mas, também afetava os profissionais que tinham menos exigência consigo mesmos e que tinham algum hobby, praticavam atividade física com regularidade, tinham mais experiência profissional e alguma especialidade (MATUBARO KCA, et al., 2009).

Identificando os principais fatores relacionados à Síndrome de Burnout e o absenteísmo entre enfermeiros que atuam no contexto hospitalar, Rezende $R$ et al. (2012) propõe que esses fatores, durante avaliação das condições de trabalho nos ambientes hospitalares sejam considerados uma vez, ainda não existem mecanismos de detecção precoce do Burnout, e para que assim, consiga se diagnosticar e intervir diante estes fatores determinantes, resultando assim, em uma melhor qualidade de vida para os enfermeiros.

\section{CONSIDERAÇÕES FINAIS}

Os estudos revelaram a notória relação entre o trabalho profissional da enfermagem e a Síndrome de Burnout, aos quais estão interligadas ao desenvolvimento de doenças ocupacionais. O acometimento desta síndrome leva ao profissional da área da saúde, em especial aos enfermeiros, a percorrerem um caminho insidioso em busca do diagnóstico, podendo causar um quadro depressivo, unidos em uma redução na qualidade de vida. Destaca-se também que a atividade do enfermeiro está sujeita a grande demanda por atenção e excessivo controle nas tarefas. Além disto, enfermeiros com maior experiência profissional são mais seguros e menos estressados, ou seja, menos suscetíveis ao Burnout. Personalidades muitos perfeccionistas e metódicas sofrem mais diante de uma frustração e podem também sofrer do esgotamento característicos do Burnout.

Conclui-se que a saúde do trabalhador de enfermagem precisa de maior atenção, devido à gravidade dos casos, e dos transtornos que esta patologia acarreta na vida dos mesmos. Todos os profissionais devem ser assegurados os seus direitos por parte das instituições de saúde e dos governos. É necessário de adotar estratégias relacionadas a diminuição das jornadas de trabalho, melhoria na infraestrutura das instituições, são exemplos de medidas profiláticas para as doenças ocupacionais como a Síndrome de Burnout. Acredita-se ser necessário que os futuros estudos visem identificar ações preventivas, contribuindo para redução das ocorrências da Síndrome de Burnout entre os enfermeiros.

\section{REFERÊNCIAS}

1. CARLOTTO MS. A síndrome de Burnout e o trabalho docente. Psicologia em Estudo, 2002; 7(1):21-9.

2. CARLOTTO MS, CÂMARA SG. Análise da produção científica sobre a Síndrome de Burnout no Brasil. PSICO, 2008; 39(2): 152-8.

3. DUARTE AP, et al. Prevalência da síndrome de Burnout em enfermeiros atuantes em UTI. Revista Portal de Periódicos Eletrônicos de Psicologia, 2013; 16: 1-12. 
4. FRANCA FM, et al. Burnout and labour aspects in the nursing teams at two medium-sized hospitals. Rev. Latino-Am. Enfermagem, 2012; 20(5): 961-70.

5. GRAZZIANO ES, BIANCHI ERF. Impacto do stress ocupacional e Burnout para Enfermeiros. Revista eletrônica semestral de enfermeira - Enfermería Global, 2010; 18: 1-20

6. GUIDO LA, et al. Estresse e Burnout entre residentes multiprofissionais. Rev. Latino-Am. Enfermagem, 2012; 20(6): 1064-71.

7. JODAS DA, HADDAD MCL. Síndrome de Burnout em trabalhadores de enfermagem de um pronto socorro de hospital universitário. Rev. Acta Paul Enferm, 2009; 22(2): 192-7.

8. LIMA FD, et al. Síndrome de Burnout em Residentes da Universidade Federal de Uberlândia. Rev. Bras. de Educ. Med., 2007; 31(2): 137-46.

9. LORENZ VR, et al. Burnout e estresse los Enfermeiros de um Hospital Universitário de Alta Complexidade. Rev. Latino-Am. Enfermagem, 2010;18(6):[08 telas].

10. MATUBARO KCA, et al. Síndrome de Burnout em profissionais de saúde: uma revisão bibliográfica. Anais do Conic-Semesp, 2013; 1: 104-12.

11. MOREIRA DS, et al. Prevalência da síndrome de burnout em trabalhadores de enfermagem de um hospital de grande porte da Região Sul do Brasil. Cad. Saúde Pública, 2009; 25(7): 1559-68.

12. REZENDE R, et al. Síndrome de Burnout e absenteísmo em enfermeiros no contexto hospitalar: revisão integrativa da literatura brasileira. Revista ESCS, 2012; 23(3): 243-52.

13. RIBEIRO RP, et al. Estresse ocupacional entre trabalhadores de saúde de um hospital universitário. Rev. Gaúcha Enferm., 2018;39:e65127

14. SOUSA MVH. Síndrome de Burnout em profissionais da saúde: estudo bibliográfico. 2012. 30p. Monografia (Bacharelado em Enfermagem), Universidade Federal do Piauí, Picos, 2012.

15. TRIGO TR, et al. Síndrome de burnout ou estafa profissional e os transtornos psiquiátricos. Rev. psiquiatr. clín., 2007; 34(5): 223-33. 